\title{
EXPLORING THE BEGINNINGS OF ALGEBRAIC K-THEORY
}

\author{
by
}

Sarah Schott

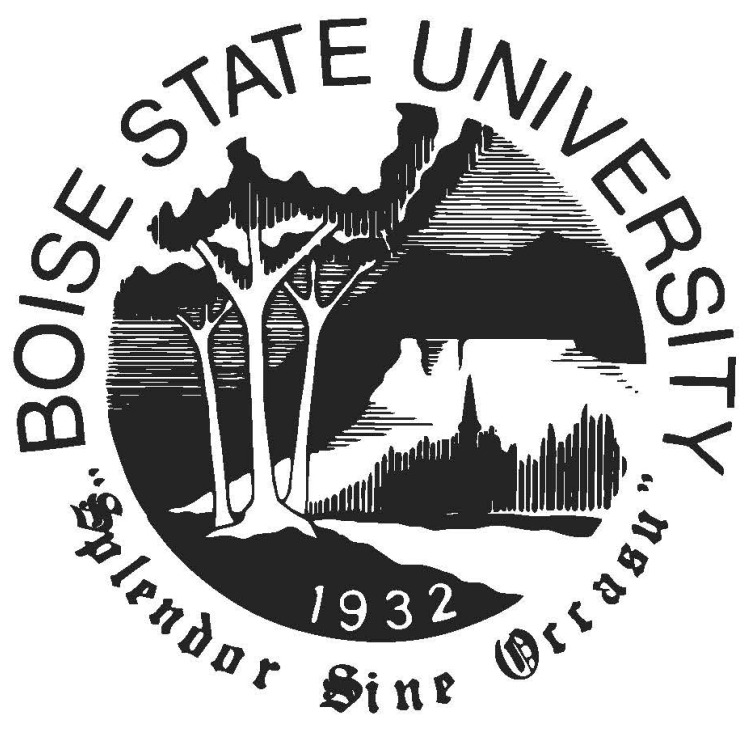

A thesis

submitted in partial fulfillment

of the requirements for the degree of

Master of Science in Mathematics

Boise State University

May 2021 
(C) 2021

Sarah Schott

ALL RIGHTS RESERVED 
BOISE STATE UNIVERSITY GRADUATE COLLEGE

DEFENSE COMMITTEE AND FINAL READING APPROVALS

of the thesis submitted by

Sarah Schott

Thesis Title: Exploring the Beginnings of Algebraic K-Theory

Date of Final Oral Examination: $\quad 03$ March 2021

The following individuals read and discussed the thesis submitted by student Sarah Schott, and they evaluated her presentation and response to questions during the final oral examination. They found that the student passed the final oral examination.

Jens Harlander, Ph.D. Chair, Supervisory Committee

Zachariah Teitler, Ph.D. Member, Supervisory Committee

Uwe Kaiser, Ph.D. Member, Supervisory Committee

The final reading approval of the thesis was granted by Jens Harlander, Ph.D., Chair of the Supervisory Committee. The thesis was approved by the Graduate College. 


\section{ACKNOWLEDGMENTS}

First and foremost, I would like to thank Jens Harlander for being my advisor and teacher. I am grateful for all of the help and guidance he gave me throughout this process. He was patient with my endless questions and always made me laugh when times were tough. I'd also like to thank Zach Teitler for acting as a mentor to me when I first arrived at Boise State. I will always appreciate his kindness and guidance. I thank Shea Vela-Vick and Robert Perlis for introducing me to topological and algebraic research at LSU. Finally, I thank my parents and friends for sticking by my side throughout my graduate career. 


\begin{abstract}
According to Atiyah, K-theory is that part of linear algebra that studies additive or abelian properties (e.g. the determinant). Because linear algebra, and its extensions to linear analysis, is ubiquitous in mathematics, K-theory has turned out to be useful and relevant in most branches of mathematics. Let $R$ be a ring. One defines $K_{0}(R)$ as the free abelian group whose basis are the finitely generated projective $R$-modules with the added relation $P \oplus Q=P+Q$. The purpose of this thesis is to study simple settings of the K-theory for rings and to provide a sequence of examples of rings where the associated $K$-groups $K_{0}(R)$ get progressively more complicated. We start with $R$ being a field or a principle ideal domain and end with $R$ being a polynomial ring on two variables over a non-commutative division ring.
\end{abstract}




\section{TABLE OF CONTENTS}

ACKNOWLEDGMENTS .................... iv

ABSTRACT $\ldots \ldots \ldots \ldots \ldots \ldots \ldots \ldots \ldots \ldots \ldots \ldots \ldots \ldots$

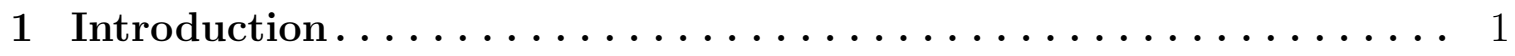

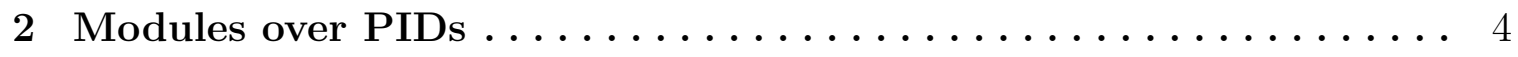

3 Projective modules over local $\operatorname{rings} \ldots \ldots \ldots \ldots \ldots \ldots$

4 Unimodular rows and stably free modules $\ldots \ldots \ldots \ldots \ldots \ldots$

5 Unimodular rows for non-commutative $\operatorname{rings} \ldots \ldots \ldots \ldots \ldots$

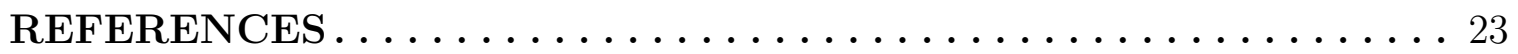




\section{CHAPTER 1}

\section{INTRODUCTION}

We quote from Atiyah's K-Theory: "K-theory is that part of linear algebra that studies additive or abelian properties (e.g. the determinant). Because linear algebra, and its extensions to linear analysis, is ubiquitous in mathematics, K-theory has turned out to be useful and relevant in most branches of mathematics."

A vector bundle over a topological space $X$ is a space $E$ together with a continuous map $p: E \rightarrow X$ so that $X$ can be covered by open sets $X=\bigcup U_{j}$ with the property $p^{-1}\left(U_{j}\right) \cong U_{j} \times \mathbb{R}^{n}$ (homeomorphic) and transitions maps are linear. Vector bundles can be added (Whitney sum) and it turns out that vector bundles are "projective": If $X$ is compact and $p: E \rightarrow X$ is a vector bundle then there exists another vector bundle $p: E^{\prime} \rightarrow X$ so that $E \oplus E^{\prime} \cong X \times \mathbb{R}^{m}$. Thus $p: E \rightarrow X$ is a projection of an obvious vector bundle $X \times \mathbb{R}^{m} \rightarrow X$. Let $K_{0}(X)$ be the free abelian group with basis the set of vector bundles with the added relation $E \oplus E^{\prime}=E+E^{\prime} . K_{0}(X)$ is an abelian group, accessible via linear algebra and analysis, that measures how tangled the space $X$ is. For example, if $X$ is contractible, then every vector bundle is of the form $X \times \mathbb{R}^{n}$ for some $n$, and so $K_{0}(X)=\mathbb{Z}$. The circle $S^{1}$ already admits vector bundles that are not products, the line bundle coming from the Möbius band for example. So $K_{0}\left(S^{1}\right) \neq \mathbb{Z}$.

Now let $R$ be a ring. An $R$-module $M$ is called projective if it is a summand of a 
free $R$-module $R^{m}$. So $M$ is a projection of an obvious $R$-module. Let $K_{0}(R)$ be the free abelian group with basis the set of projective $R$-modules with the added relation $M \oplus M^{\prime}=M+M^{\prime} . K_{0}(R)$ is an abelian group that reflects the complexity of the ring $R$. For example if $R$ is a field, then every projective $R$-module is of the form $R^{n}$ for some $n$, and so $K_{0}(R)=\mathbb{Z}$.

The purpose of this thesis is to study simple settings of the K-theory for rings and to provide a sequence of examples of rings where $K_{0}(R)$ gets progressively more complicated. A unimodular row is a surjective linear map $\alpha: R^{m} \rightarrow R$. The kernel ker $\alpha$ is a projective $R$-module that may or may not be projective. We show:

1. The kernel ker $\alpha$ of an unimodular row $\alpha: R^{m} \rightarrow R$ is free if and only if it can be extended to an invertible $m \times m$-matrix.

2. If $R$ is a PID, then every unimodular row can be extended to an invertible matrix. In fact, every finitely generated $R$-module is free.

3. If $R$ is a local ring, then every unimodular row can be extended to an invertible matrix. In fact, every projective $R$-module is free. However, there do exist local rings where finitely generated modules are not always free.

4. If $R$ is a commutative ring and the unimodular row has length 2 , then it can be extended to an invertible $2 \times 2$-matrix. However, there do exist commutative rings so that a unimodular row of length 3 can not be extended to an invertible $3 \times 3$-matrix. In particular there exists projective $R$-modules that are not free.

5. For every non-commutative division ring $D$ there does exists a length 2 unimodular row over the polynomial ring $R=D[x, y]$ that can not be extended to an invertible $2 \times 2$-matrix. 
The main sources for this thesis are the first chapter in Weibel's K-Book [7] Rotman's "Advanced Modern Algebra" [5], Daniel Chan's video on vector bundles [1], Wikipedia's article on Algebraic K-theory [8], Jason Polak's blog post on projective modules over local rings [4], and Nicholson's book "Introduction to Abstract Algebra" [3]. 


\section{CHAPTER 2}

\section{MODULES OVER PIDS}

The main source for this chapter is Rotman, Advanced Modern Algebra [5]

An $R$-module has the same definition as a vector space, except scalars are in a ring $R$ instead of a field.

Definition 2.0.1. Let $R$ be ring with an identity element 1 . A left $R$-module is an (additive) abelian group $M$ equipped with a scalar multiplication such that the following axioms hold for all $m, m^{\prime} \in M$ and all $r, r^{\prime}, 1 \in R$ :

(i) $r\left(m+m^{\prime}\right)=r m+r m^{\prime}$

(ii) $\left(r+r^{\prime}\right) m=r m+r^{\prime} m$

(iii) $\left(r r^{\prime}\right) m=r\left(r^{\prime} m\right)$

(iv) $1 m=m$

Definition 2.0.2. A $\boldsymbol{P I D}$ (Principal Ideal Domain) is an integral domain where every proper ideal can be generated by a single element.

\section{Examples of PIDs:}

(i) The ring of integers

(ii) Any field

(ii) Euclidean rings 


\section{Examples of Rings which are not PIDs:}

(i) $\mathbb{Z}[x]$

(ii) $\mathbb{Q}[x, y]$

Definition 2.0.3. An $R$-module $F$ is called a free $\boldsymbol{R}$-module if there exists a linearly independent generating set $B=\left\{b_{i}: i \in I\right\}$, called a basis. Note that in that case $F$ is isomorphic to a direct sum of copies of $R$ : The isomorphism

$$
F \rightarrow \bigoplus_{i \in I} R_{i}
$$

send a linear combination $\sum_{i \in I} r_{i} b_{i}$ to the tupel $\left(r_{i}\right)_{i \in I}$.

Lemma 2.0.4. Suppose we have an epimorphism $f: M \rightarrow F$ where $M$ is an $R$ module and $F$ is a free $R$-module. Then there exists a splitting $g: F \rightarrow M(f \circ g=i d)$ and the homomorphism $h: M \rightarrow F \oplus \operatorname{ker}(f)$ defined by $h(m)=(f(m), m-g \circ f(m))$ is an isomorphism.

Proof. Since $F$ is free, it has a basis: $b_{1}, \ldots, b_{k}$. We know that $f$ is onto so choose $m_{1}, \ldots, m_{k}$ so that $f\left(m_{i}\right)=b_{i}$. Thus, we can define a homomorphism $g: F \rightarrow M$ by $g\left(b_{i}\right)=m_{i}$ and it follows that $f\left(g\left(b_{i}\right)\right)=f\left(m_{i}\right)=b_{i}$. Now, $h$ is a homomorphism. Let us consider the coordinates in the image of $h . f$ is an epimorphism, so it is a homomorphism. Further, the composition of two homomorphisms is a homomorphism, so $m \mapsto m-g \circ f(m)$ is also a homomorphism. We now show that the homomorphism $h$ is an isomorophism. First, suppose $h(m)=(0,0)$. Then clearly $f(m)=0$ and so we have $m-g(0)=0$. Since $g$ is a homomorphism we have $g(0)=0$ and can conclude that $m=0$, and therefore $h$ is injective. To show that $h$ is onto, we must show that 
given $(p, q) \in F \oplus \operatorname{ker}(f)$ there exists an $m$ so that $h(m)=(p, q)$. Let $m:=g(p)+q$. Then it follows:

$$
\begin{aligned}
f(m) & =f(g(p)) \\
& =p
\end{aligned}
$$

From this we see that

$$
\begin{aligned}
m-g(f(m)) & =g(p)+q-g(p) \\
& =q
\end{aligned}
$$

So we have

$$
\begin{aligned}
h(m) & =(f(m), m-g(f(m)) \\
& =(p, q)
\end{aligned}
$$

Definition 2.0.5. Let $M$ be an $R$-module. The annihilator of $m \in M$ is defined as the following: ann $m=\{r \in R: r m=0\}$.

Definition 2.0.6. An element $m \neq 0$ in an $R$-module $M$ is called a torsion element if ann $m \neq 0$. An $R$-module $M$ is torsion-free if it does not contain torsion elements. $M$ is a torsion module if every nonzero element in $M$ is a torsion element.

Definition 2.0.7. If $N$ is a submodule of an $R$-module $M$, then the quotient module is the quotient group $M / N$ equipped with the scalar multiplication

$$
r(m+N)=r m+N
$$


Theorem 2.0.8. (Rotman [5])If $R$ is a PID, then every finitely generated torsion-free $R$-module $M$ is free.

Proof. The theorem is proven by induction on the number of generators of $M$. So suppose $M$ is a torsion-free $R$-module generated by the set of non-zero elements $\left\{v_{1}, \ldots, v_{n}\right\}$. Each $m \in M$ has the form $m=r_{1} v_{1}+\ldots+r_{n} v_{n}$ where the $r_{i}$ 's are elements of $R$. For the base case, assume $M=\left\langle v_{1}\right\rangle$, which means that if $m \in M$, then $m=r v_{1}$ for some $r \in R$. Since $M$ was assumed to be torsion-free and $v_{1} \neq 0$, $r v_{1}=0$ implies $r=0$. This shows independence, hence $\left\{v_{1}\right\}$ is a basis for $M$.

For the inductive step, let $M=\left\langle v_{1}, \ldots, v_{n+1}\right\rangle$ and define

$$
S=\left\{m \in M: \text { there is } r \in R, r \neq 0, \text { with } r m \in\left\langle v_{n+1}\right\rangle\right\}
$$

First, we check that $S$ is a submodule of $M$. To show closure under addition, suppose we have two elements of $S: m_{1}, m_{2}$. Then we have the following:

$$
\begin{gathered}
r_{1} m_{1}=r_{1}^{\prime} v_{n+1} \\
r_{1} r_{2} m_{1}=r_{2} r_{1}^{\prime} v_{n+1}
\end{gathered}
$$

and we have:

$$
\begin{gathered}
r_{2} m_{2}=r_{2}^{\prime} v_{n+1} \\
r_{1} r_{2} m_{2}=r_{1} r_{2}^{\prime} v_{n+1}
\end{gathered}
$$

Adding the equations together, we see that $r_{1} r_{2}\left(m_{1}+m_{2}\right)=\left(r_{2} r_{1}^{\prime}+r_{1} r_{2}^{\prime}\right) v_{n+1}$. Thus, $S$ is closed under addition. Furthermore, if $m \in S$, then $r^{\prime} m=r^{\prime \prime} v_{n+1}$ where $r^{\prime}, r^{\prime \prime} \in R$. Multiplying both sides of the equation by $r$ we have: $r^{\prime} r m=r r^{\prime \prime} v_{n+1}$. Since $r r^{\prime} \in R$, we can conclude that $S$ is closed under scalar multiplication. 
Now, to show that $M / S$ is torsion-free, we assume otherwise and arrive at a contradiction. So suppose $x \in M, x \notin S$, and $r(x+S)=0+S=S$, with $r \neq 0$. Then we have the following:

$$
\begin{array}{r}
r(x+S)=S \\
r x+S=S
\end{array}
$$

Thus, $r x \in S$. Since this is the case, there exists an $r^{\prime} \in R$ with $r^{\prime} \neq 0$ and $r r^{\prime} x \in\left\langle v_{n+1}\right\rangle$. Since $r r^{\prime} \neq 0$, we have $x \in S$, a contradiction. So $M / S$ is torsion-free. Now we have the following:

$$
\begin{aligned}
m+S & =\left(r_{1} v_{1}+\ldots+r_{n} v_{n}\right)+S \\
& =r_{1} v_{1}+S+\ldots+r_{n} v_{n}+S \\
& =r_{1}\left(v_{1}+S\right)+\ldots+r_{n}\left(v_{n}+S\right)
\end{aligned}
$$

So $M / S$ is generated by $n$ elements. Thus, $M / S$ is free by the inductive hypothesis. Note that we have a projection map $M \rightarrow M / S$ with kernel $S$. So it follows from Lemma 2.0.4 that

$$
M \cong S \oplus(M / S)
$$

Since the direct sum of free modules is free, all we have left to show is that $S \cong R$. If $x \in S$, then there is some nonzero $r \in R$ with $r x \in\left\langle v_{n+1}\right\rangle$; that is, there exists $a \in R$ with $r x=a v_{n+1}$. Define $\varphi: S \rightarrow Q=\operatorname{Frac}(R)$ by $\varphi: x \mapsto \frac{a}{r}$. Let $r_{1} x=a_{1} v_{n+1}$ and $r_{2} x=a_{2} v_{n+1}$. Then $x=\frac{a_{1}}{r_{1}} v_{n+1}$ and $x=\frac{a_{2}}{r_{2}} v_{n+1}$, so it follows that $\frac{a_{1}}{r_{1}}=\frac{a_{2}}{r_{2}}$. Thus, $\varphi$ is well-defined. Suppose $x_{1}, x_{2} \in S$ and $\varphi\left(x_{1}\right)=\varphi\left(x_{2}\right)$. Then we have 


$$
\begin{aligned}
r_{1} x_{1} & =a_{1} v_{n+1} \\
r_{2} x_{2} & =a_{2} v_{n+1} \\
\frac{a_{1}}{r_{1}} & =\frac{a_{2}}{r_{2}}
\end{aligned}
$$

If $r=r_{1} r_{2}, a=a_{1} r_{2}=a_{2} r_{1}$, then it follows that $r x_{1}=a v_{n+1}$ and $r x_{2}=a v_{n+1}$. We have

$$
\begin{aligned}
r x_{1} & =r x_{2} \\
r\left(x_{1}-x_{2}\right) & =0 \\
x_{1}-x_{2} & =0 \\
x_{1} & =x_{2}
\end{aligned}
$$

Thus, $\varphi$ is injective. If $D=\operatorname{im} \varphi$, then $D$ is a finitely generated submodule of $Q$. ( $D$ is finitely generated because it is the image of $S$, and $S$ is finitely generated because $S$ is a direct summand of the finitely generated module $M$ ) We know that $D$ is a submodule of $Q$ because if $\frac{a}{b}, \frac{c}{d} \in D$, then clearly their sum is in $D$ as well. It is also clear that if $\frac{a}{b} \in D$, and $r \in R, r \cdot \frac{a}{b}$ is in $D$. Now suppose

$$
D=\left\langle\frac{b_{1}}{c_{1}}, \ldots \frac{b_{m}}{c_{m}}\right\rangle
$$

where $b_{i}, c_{i} \in R$. Let $c=\prod_{i} c_{i}$ and define $f: D \rightarrow R$ by $f: d \mapsto c d$ for all $d \in D$. It is clear that $f$ has values in $R$ because for each $d \in D$ we have $d=r_{1} \frac{b_{1}}{c_{1}}+\ldots r_{m} \frac{b_{m}}{c_{m}}$ and $c=c_{1} \cdot c_{2} \cdot \ldots \cdot c_{m} \cdot \ldots$, so multiplying $d$ by $c$ clears all denominators. Since $D$ is torsion-free, $f$ is an injective $R$-map, and so $D$ is isomorphic to an ideal of $R$. Since $R$ is a PID, every nonzero ideal in $R$ is isomorphic to $R$; hence, $S \cong \operatorname{im} f=D \cong R$. Since $S$ is isomorphic to $R$, it follows that $S$ is free and since $M / S$ is free, $M \cong S \oplus(M / S)$ is free as well. 


\section{CHAPTER 3}

\section{PROJECTIVE MODULES OVER LOCAL RINGS}

The main result in this chapter is due to Kaplansky [2]. Our discussion follows the blog entry of Jason Polak [4].

Definition 3.0.1. A local ring is a ring in which the set of nonunits forms an ideal.

Note that this ideal of nonunits is the unique maximal ideal in the ring. In fact, local rings are exactly the rings with unique maximal ideals. A local ring does not have to be commutative.

\section{Examples of Local Rings:}

(i) All fields are local rings since the only non-unit, $\{0\}$, forms an ideal.

(ii) The ring of rational numbers with odd denominator is local. The set of non-units consist of the fractions with an even numerator and an odd denominator.

Definition 3.0.2. An $R$-module is projective it is a direct summand of a free module.

It is clear that a direct summand of a projective module is projective.

\section{An Example of a Projective Module Which is Not Free:}

It is well known that projective modules need not be free. As an example, consider the abelian group: $\mathbb{Z}_{6}=\mathbb{Z}_{3} \oplus \mathbb{Z}_{2}$. Since $\mathbb{Z}_{6}$ is a free $\mathbb{Z}_{6}$-module, (basis $\{\overline{1}\}$ or $\{\overline{5}\}$, it follows that $\mathbb{Z}_{3}$ is a projective $\mathbb{Z}_{6}$-module. Now $\mathbb{Z}_{3} \oplus\{0\}=\{(0,0),(1,0),(2,0)\}$ has three elements. Let's say that each free $\mathbb{Z}_{6}$ module has a basis with $n$ elements. 
A finitely-generated free $\mathbb{Z}_{6}$-module is a direct sum of $n$ copies of $\mathbb{Z}_{6}$, so any free $\mathbb{Z}_{6}$-module has $6^{n}$ elements. Hence, $\mathbb{Z}_{3} \oplus\{0\}$ is a projective $\mathbb{Z}_{6}$-module that is not free.

Corollary 3.0.3. Let $S$ be a finite ring. Then $S \oplus\{0\}$ is a projective $S \oplus S$-module that is not free.

Proof. Suppose that $S=\left\{r_{1}, \ldots, r_{n}\right\}$. Then $S \oplus S$ has $n^{2}$ elements. Also suppose that $\left\{x_{1}, \ldots, x_{k}\right\}$ is a basis for $S \oplus\{0\}$. Then $S \oplus\{0\}=(S \oplus S) x_{1} \oplus \ldots \oplus(S \oplus S) x_{k}$ and is a direct sum of $k$ copies of $S \oplus S$. So any free $S \oplus S$-module has $\left(n^{2}\right)^{k}$ elements. Hence, $S \oplus\{0\}$ is not a free $S \oplus S$-module.

Lemma 3.0.4. Let $R$ be a ring and $M$ a finitely generated $R$-module. Suppose that any direct summand $N$ of $M$ has the following property: for any element $x \in N$, there exists a free direct summand $F$ of $N$ such that $x \in F$. Then $M$ is free.

Proof. . Let $\left\{x_{1}, \ldots, x_{n}\right\}$ be a generating set for $M$. Since $M$ is a direct summand of $M$ thus there exists a decomposition $M=F_{1} \oplus M_{1}$ with $x_{1} \in F_{1}$. Let $y_{i}$ be the projection of $x_{i}$ to $M_{1}$. Then $\left\{y_{2}, \ldots, y_{n}\right\}$ is a generating set for $M_{1}$. Some of the $y_{i}$ might be zero, throw them out. If they are all zero then $M=F_{1}$ and we are done. Assume without loss of generality that $y_{2}$ is not zero. Then there exists a decomposition $M_{1}=F_{2} \oplus M_{2}$ so that $y_{2} \in F_{2}$. We proceed in this fashion and in the end arive at $M=F_{1} \oplus F_{2} \oplus \cdots \oplus F_{k}$. So $M$ is free.

This Lemma has the following consequence.

Lemma 3.0.5. Let $R$ be a ring and suppose that every finitely generated projective $R$-module $P$ has the following property: for any element $x \in P$ there exists a free summand of $P$ that contains $x$. Then all finitely generated projectives are free. 
Proof. Let $P$ be a finitely generated projective. Let $N$ be a summand and $x \in N$. Then $N$ is a finitely generated projective and hence $N$ contains a free summand that contains $x$. Thus $P$ satisfies Lemma 3.0.4. So $P$ is free.

Theorem 3.0.6. (Kaplansky [2]) A projective module over a local ring is free.

Proof. We show the theorem only for finitely generated projectives $P$. Let $x \in P$. By Lemma 3.0.5 it suffices to construct a free summand $S$ of $P$ that contains $x$. Because $P$ is projective, we can write $F=P \oplus Q$ where $F$ is free. Choose a basis $\left\{u_{i}\right\}$ of $\mathrm{F}$ so that the number of generators required to express $\mathrm{x}$ is minimal, and write

$$
x=a_{1} u_{1}+\ldots+a_{n} u_{n}
$$

Because of minimality no $a_{i}$ in this sum can be expressed in terms of the other $a_{j}$. For suppose the simplest case, $x=a_{1} u_{1}+a_{2} u_{2}$ and $a_{2}=r a_{1}$. Then $x=a_{1}\left(u_{1}+r u_{2}\right)$. We can now switch to the basis $u_{1}^{\prime}=u_{1}+r u_{2}, u_{2}, u_{3}, \ldots$, and in that basis $x=a_{1} u_{1}^{\prime}$, which contradicts minimality. Let $y_{i}$ be the image of $u_{i}$ under the projection $F \rightarrow P$. We have

$$
x=a_{1} u_{1}+\ldots+a_{n} u_{n}=a_{1} y_{1}+\ldots+a_{n} y_{n}
$$

Now write $y_{i}=\left(\sum_{j=1}^{n} c_{i j} u_{j}\right)+t_{i}$ where $t_{i}$ is a linear combination of the remaining basis elements $u_{j}$ not in $\left\{u_{1}, \ldots, u_{n}\right\}$. We have $y_{i}-t_{i}=\sum_{j=1}^{n} c_{i j} u_{j}$. If we can show that the $n \times n$-matrix $\left(c_{i j}\right)$ is invertible, then the $y_{i}-t_{i}$ together with the $u_{j}, j \neq 1, \ldots, n$ is a basis for $F$. And therefore the $y_{i}$ together with the $u_{j}, j \neq 1, \ldots, n$ is a basis for $F$. Then the $y_{i}$ are a basis for a free summand $S$ of $F$ (and hence of $P$ ) that contains $x$ and we are done.

If we input the $y_{i}$ into the equation $a_{1} u_{1}+\ldots+a_{n} u_{n}=a_{1} y_{1}+\ldots+a_{n} y_{n}$ and it is easy to see that 


$$
a_{j}=a_{1} c_{1 j}+\ldots+a_{n} c_{n j}
$$

Rearranging the above equation, we see that $\left(1-c_{j j}\right)$ is a non-unit. Indeed, we have

$$
\left(1-c_{j j}\right) a_{j}=a_{j}-c_{j j} a_{j j}=a_{1} c_{1 j}+\ldots a_{j-1} c_{j-1 j}+a_{j+1} c_{j+1 j}+\cdots+a_{n} c_{n j} .
$$

So if $\left(1-c_{j j}\right)$ were a unit, we could divide and express $a_{j}$ in terms of the other $a$ 's, a contradiction to minimality (see the first paragraph of the proof). It follows that since $\left(1-c_{j j}\right)$ is a non-unit, $c_{j j}$ must be a unit. Here we use that our ring $R$ is local. In a similar way, we conclude that $c_{i j}$ is a non-unit. Thus, we have a matrix with units on the diagonal and non-units off the diagonal. Such a matrix over a local ring is invertible. In order to see this decompose the $C$ into $C=D+O D$, where $D$ is diagonal part of $C$ and $O D$ is the off-diagonal part. To see that $C$ is invertible, we use the Jacobson ideal and its properties. See Rotman [5]. The Jacobson radical $J(R)$ of a ring is the intersection of all maximal ideals. So if $R$ is local then $J(R)$ are the nonunits. In general we have that $J(M(n, R))=M(n, J(R))$. Now look at $C=D+O D$. We have $O D \in M(n, J(R))$ and so $O D \in J(M(n, R))$. Thus $C+J(M(n, R))=D+J(M(n, R))$. Since $D$ is a unit, $C$ has to be one as well (see Rotman [5] page 544, Proposition 8.3). 


\section{CHAPTER 4}

\section{UNIMODULAR ROWS AND STABLY FREE MODULES}

The main source for this chapter is Weibel's K-book [7].

Let $R$ be a ring with identity that satisfies the rank invariance property. $R$-modules are right $R$-modules. Let $\alpha: R^{m} \rightarrow R^{n}$ be an onto map $(m \geq n)$. We say $\hat{\alpha}: R^{m} \rightarrow$

$R^{n} \oplus R^{m-n}$ is an isomorphic extension of $\alpha$ if $\hat{\alpha}$ is an isomorphism and $p \circ \hat{\alpha}=\alpha$, where $p$ is the projection on the first $n$ coordinates.

Theorem 4.0.1. Let $\alpha: R^{m} \rightarrow R^{n}$ be an onto module homomorphism $(m \geq n)$. Then $\alpha$ has an extension $\hat{\alpha}$ if and only if $\operatorname{ker} \alpha$ is free of rank $m-n$

Proof. Let us assume first that $\alpha$ has an isomorphic extension $\hat{\alpha}$. Note that $\operatorname{ker} \alpha=$ $\operatorname{ker}(p \circ \hat{\alpha})=\hat{\alpha}^{-1}\left(R^{m-n}\right)$. So ker $\alpha$ is free of rank $m-n$.

Next suppose that $M=\operatorname{ker} \alpha$ is free of rank $m-n$. Choose an isomorphism $\beta: M \rightarrow$ $R^{m-n}$ and a splitting $s: R^{n} \rightarrow R^{m}$ of $\alpha$. We have $\alpha \circ s=1$. Note that

$$
\begin{aligned}
\alpha(\vec{r}-s(\alpha(\vec{r}))) & =\alpha(\vec{r})-\alpha(s(\alpha(\vec{r}))) \\
& =\alpha(\vec{r})-\alpha(\vec{r}) \\
& =0
\end{aligned}
$$

so $\vec{r}-s(\alpha(\vec{r})) \in \operatorname{ker} \alpha$. It follows that every element in $R^{m}$ can be uniquely written as $\vec{r}=s \circ \alpha(\vec{r})+(\vec{r}-s \circ \alpha(\vec{r}))$, so $R^{m}=s\left(R^{n}\right) \oplus M$. Now define $\hat{\alpha}: R^{m} \rightarrow R^{n} \oplus M \rightarrow$ 
$R^{n} \oplus R^{m-n}$ by $\hat{\alpha}(\vec{r})=\alpha(\vec{r})+\beta(\vec{r}-s \circ \alpha(\vec{r}))$. This is an isomorphism with the desired property.

Here is the matrix version of the above theorem.

Theorem 4.0.2. Let $\alpha$ be a $n \times m$ matrix ( $n$ rows and $m$ columns), $m \geq n$, that defines a surjection $\alpha: R^{m} \rightarrow R^{n}$ with kernel $M$. Then $M$ is isomorphic to $R^{m-n}$ if and only if $\alpha$ can be extended to an invertible $m \times m$ matrix $\hat{\alpha} \in G L(m, R)$.

The $(m-n) \times m$ matrix $\alpha^{\prime}: R^{m} \rightarrow R^{m-n}$ which, together with the matrix $\alpha$ gives $\hat{\alpha}$ (in case $\operatorname{ker} \alpha$ is free) is given by $\alpha^{\prime}(\vec{r})=\beta(\vec{r}-s \circ \alpha(\vec{r}))$. Note that $\hat{\alpha}$ is obtained by stacking $\alpha$ and $\alpha^{\prime}$ on top of each other.

Corollary 4.0.3. If $R$ is a field then an $n \times m$-matrix $\alpha, m \geq n$, of rank $n$ can be extended to an invertible $m \times m$-matrix $\hat{\alpha}$. The $(m-n) \times m$ matrix $\alpha^{\prime}$ that extends $\alpha$ to $\hat{\alpha}$ can be chosen so that every row of $\alpha^{\prime}$ is orthogonal to every row of $\alpha$.

Proof. We give an independent elementary proof. Let $\overrightarrow{a_{1}}, \ldots, \overrightarrow{a_{n}}$ be the rows of $\alpha$. Since the rank of $\alpha$ is $n$ we know that these rows are linearly independent. Note that $R^{m}=(\operatorname{ker} \alpha)^{\perp} \oplus \operatorname{ker} \alpha$ and that $(\operatorname{ker} \alpha)^{\perp}$ is the row space of $\alpha$ with basis $\overrightarrow{a_{1}}, \ldots, \overrightarrow{a_{n}}$. Let $\overrightarrow{a_{m-n+1}^{\prime}}, \ldots, \overrightarrow{a_{m}^{\prime}}$ be a basis for ker $\alpha$. Then the rows $\overrightarrow{a_{1}}, \ldots, \overrightarrow{a_{n}}$ together with the rows $a_{m-n+1}^{\prime}, \ldots, \overrightarrow{a_{m}^{\prime}}$ form a set of $m$ linearly independent vectors. So they make up an invertible $m \times m$-matrix.

A unimodular row is a matrix $\left[a_{1}, \ldots, a_{m}\right]$ consisting of a single row that defines a surjection $R^{m} \rightarrow R$.

Theorem 4.0.4. Let $[a b]$ be a unimodular row over a commutative ring $R$. Then it can be extended to an invertible $2 \times 2$ matrix. 
Proof. If $\left[\begin{array}{ll}a & b\end{array}\right]$ is a unimodular row then there exists $c, d \in R$ such that $a c+b d=1$. Thus, we can extend $\left[\begin{array}{ll}a & b\end{array}\right]$ to $\left[\begin{array}{cc}a & b \\ -d & c\end{array}\right]$, which is invertible because its determinant is 1 .

This is not true for longer unimodular rows: there does exist a unimodular row of length 3 over a commutative ring that does not extend to an invertible $3 \times 3$ matrix. Before we give an example we recall some algebraic topology.

A tangent vector field on $S^{n}$ is a continuous map $v: S^{n} \rightarrow \mathbb{R}^{n+1}$ so that the dot product $x \cdot v(x)=0$, i.e. $x$ and $v(x)$ are orthogonal.

Theorem 4.0.5. $S^{n}$ admits a non-zero tangent vector field if and only if $n$ is odd.

For a proof see Hatcher, Theorem 2.28, page 135. We are now ready to give our example. Let $R=\mathbb{R}[x, y, z] /\left(x^{2}+y^{2}+z^{2}=1\right)$, a quotient of a polynomial ring. Note that $\alpha=\left[\begin{array}{lll}x & y & z\end{array}\right]$ is a unimodular row because

$$
x x+y y+z z=1
$$

Suppose this row could be extended to a invertible $3 \times 3$ matrix

$$
\left[\begin{array}{ccc}
x & y & z \\
f_{21} & f_{22} & f_{23} \\
f_{31} & f_{32} & f_{33}
\end{array}\right]
$$

where the $f_{i j}$ are polynomials in $x, y, z$, considered modulo the given ideal. Let 


$$
V: S^{2} \rightarrow \mathbb{R}^{3}
$$

defined by $V(a, b, c)=\pi\left(f_{21}(a, b, c), f_{22}(a, b, c), f_{23}(a, b, c)\right)$, where $\pi: \mathbb{R}^{3} \rightarrow \mathbb{R}^{3}$ is the projection onto the orthogonal complement of $(a, b, c)$. Since the vectors $(a, b, c)$ and $\left(f_{21}(a, b, c), f_{22}(a, b, c), f_{23}(a, b, c)\right)$ are linearly independent for every $(a, b, c)$, this is a non-zero tangent vector field on $S^{2}$, contradicting Theorem 4.0.5

Remark. The tangent bundle $\mathrm{T}\left(\mathrm{S}^{2}\right)$ on the 2-sphere $S^{2}$ is a non-trivial 2-dimensional real vector bundle. If we add the normal line bundle we get the trivial bundle. The connection between $T\left(S^{2}\right)$ and the non-free projective module we considered (the kernel of the unimodular row $\left.\left[\begin{array}{lll}x & y & z\end{array}\right]\right)$ is made explicit in Swan [6], Example 1. 


\section{CHAPTER 5}

\section{UNIMODULAR ROWS FOR NON-COMMUTATIVE RINGS}

The next example illustrates that there are unimodular rows of length 2 over noncommutative rings whose kernels are not free. See Exercise 1.6 in Weibel's K-book $[7]$.

Consider the following: Let $D$ be a division ring which is not a field. Choose $\alpha, \beta \in$ $D$ such that $\alpha \beta-\beta \alpha \neq 0$. Note first that $\sigma=[x+\alpha y+\beta]$ is a unimodular

row over $R=D[x, y]$ : We must show that there exists $d_{1}, d_{2} \in D[x, y]$ such that $x+\alpha) d_{1}+(y+\beta) d_{2}=1$. Consider the following combination:

$$
\begin{aligned}
(x+\alpha)(y+\beta)-(y+\beta)(x+\alpha) & =x y+\beta x+\alpha \beta+\alpha y-y x-\alpha y-\beta x-\beta \alpha \\
& =\alpha \beta-\beta \alpha
\end{aligned}
$$

Since we assumed $\alpha \beta-\beta \alpha \neq 0$, we can divide both sides of the equation by $\alpha \beta-\beta \alpha$ to arrive at our intended result. Therefore, there exists $d_{1}, d_{2} \in D[x, y]$ such that $(x+\alpha) d_{1}+(y+\beta) d_{2}=1: d_{1}=\frac{(y+\beta)}{\alpha \beta-\beta \alpha}$ and $d_{2}=\frac{-(x+\alpha)}{\alpha \beta-\beta \alpha}$.

Let $P=\operatorname{ker}(\sigma)$. We have $P \oplus R \cong R^{2}$ by Lemma 2.0.4. So $P$ is a rank 1 stably free projective. 
Theorem 5.0.1. $P$ is not a free $R$-module.

Lemma 5.0.2. $P$ does not contain a vector $\left(\begin{array}{l}f \\ g\end{array}\right)$ where both $f$ and $g$ are constant or both are linear.

Proof. Let $f=c_{1}+c_{2} x+c_{3} y$ and $g=d_{1}+d_{2} x+d_{3} y$. Set up the equation:

$$
(x+\alpha)\left(c_{1}+c_{2} x+c_{3} y\right)+(y+\beta)\left(d_{1}+d_{2} x+d_{3} y\right)=0
$$

Solving for the coefficients yields $c_{2}=d_{3}=0$ and so we have the following equations:

$$
\begin{array}{r}
c_{1}+\beta d_{2}=0 \\
c_{3}+d_{2}=0 \\
\alpha c_{3}+d_{1}=0 \\
\alpha c_{1}+\beta d_{1}=0
\end{array}
$$

Doing the appropriate substitutions we obtain the following:

$$
\begin{aligned}
& c_{1}=-\beta d_{2} \\
& c_{1}=-\alpha^{-1} \beta d_{1} \\
& c_{3}=-d_{2} \\
& c_{3}=-\alpha^{-1} d_{1}
\end{aligned}
$$

Setting the $c_{1}^{\prime} s$ equal we see that $d_{1}=\beta^{-1} \alpha \beta d_{2}$. Substituting $d_{1}$ into the $c_{3}$ equation, we see that $c_{3}=-\alpha^{-1}\left(\beta^{-1} \alpha \beta\right) d_{2}$. Finally, we set this $c_{3}$ equal to $-d_{2}$ and obtain a contradiction: $\alpha \beta=\beta \alpha$. Thus, $f$ and $g$ cannot both be linear. 
Lemma 5.0.3. $P$ does contain a vector $\left(\begin{array}{l}f \\ g\end{array}\right)$ where both $f$ and $g$ are quadratic without constant term: $f=c_{1} x+c_{2} y+c_{3} x y+c_{4} y^{2}$ and $g=d_{1} x+d_{2} y+d_{3} x y+$ $d_{4} x^{2},\left(c_{i}, d_{i} \in D\right)$.

Proof. Start by solving the following equation:

$$
(x+\alpha)\left(c_{1} x+c_{2} y+c_{3} x y+c_{4} y^{2}\right)+(y+\beta)\left(d_{1} x+d_{2} y+d_{3} x y+d_{4} x^{2}\right)=0
$$

You will then get the following equations from the coefficients:

$$
\begin{aligned}
c_{1}+\beta d_{4} & =0 \\
c_{2}+\alpha c_{3}+d_{1}+\beta d_{3} & =0 \\
c_{3}+d_{4} & =0 \\
c_{4}+d_{3} & =0 \\
\alpha c_{1}+\beta d_{1} & =0 \\
\alpha c_{2}+\beta d_{2} & =0 \\
\alpha c_{4}+d_{2} & =0
\end{aligned}
$$

Making some substitutions and letting $d_{2}=u$ we obtain the following solutions: 


$$
\begin{aligned}
& c_{1}=-\beta d_{4} \\
& c_{2}=-\alpha^{-1} \beta u \\
& c_{3}=-d_{4} \\
& c_{4}=-\alpha^{-1} u \\
& d_{1}=\beta^{-1} \alpha \beta d_{4} \\
& d_{2}=\left(\alpha^{-1} \beta-\beta \alpha^{-1}\right)^{-1}\left(-\alpha+\beta^{-1} \alpha \beta\right) d_{4} \\
& d_{3}=\alpha^{-1} u \\
& d_{4}=d_{4}
\end{aligned}
$$

Lemma 5.0.4. $P$ does contain a vector $\left(\begin{array}{l}f \\ g\end{array}\right)$ where both $f$ and $g$ contains a constant term. In fact, $f$ and $g$ can be chosen to have the special form

$$
f=\gamma_{0}+\gamma_{1} y+y^{2}, g=\delta_{0}+\delta_{1} x-\alpha y-x y
$$

and both $\gamma_{0}=\beta u^{-1} \beta u$ and $\delta_{0}$ are nonzero.

Proof. We first show that $P$ contains a vector of the form given. First, we solve the equation:

$$
(x+\alpha)\left(\gamma_{0}+\gamma_{1} y+y^{2}\right)+(y+\beta)\left(\delta_{0}+\delta_{1} x-\alpha y-x y\right)=0
$$

We obtain the following equations: 


$$
\begin{gathered}
\gamma_{0}+\beta \delta_{1}=0 \\
\gamma_{1}+\delta_{1}=\beta \\
\alpha \gamma_{1}+\delta_{0}=\beta \alpha \\
\alpha \gamma_{0}+\beta \delta_{0}=0
\end{gathered}
$$

By substitution we have the following solution for $\gamma_{0}$ :

$$
\gamma_{0}=\beta\left(-(\beta \alpha-\alpha \beta)^{-1} \cdot \beta(-(\beta \alpha-\alpha \beta))\right.
$$

So indeed $\gamma_{0}$ is a constant. Completing some substitutions in the above equations, we see that $\delta_{0}=\beta \alpha-\alpha\left(\beta-\left(-\beta^{-1} \gamma_{0}\right)\right)$, which again is a constant.

We can now prove Theorem 5.0.1. Assume $P$ is free, say $P=R^{n}$. Since $P \oplus R \cong$ $R^{2}$ it follows that $R^{n} \oplus R \cong R^{2}$ and hence $n=1$ ( $R$ has the rank invariance property). So $P$ is cyclic. Say it is generated by $\left(\begin{array}{l}r \\ s\end{array}\right)$. Let $\left(\begin{array}{l}f \\ g\end{array}\right)$ be a vector where $f$ and $g$ are both quadratics without constant terms as in Lemma 5.0.3. We have

$$
\left(\begin{array}{l}
f \\
g
\end{array}\right)=\left(\begin{array}{l}
r \\
s
\end{array}\right) h
$$

for some $h \in R$. So $2=\operatorname{deg} f=\operatorname{deg} r+\operatorname{deg} h$ and it follows that $\operatorname{deg} r=2-\operatorname{deg} h$. Similarly $\operatorname{deg} s=2-\operatorname{deg} h$. From Lemma 5.0.2 it follows that $\operatorname{deg} h=0$ and so $\operatorname{deg} r=\operatorname{deg} s=2$. So $0 \neq h \in D$ and it follows that $\left(\begin{array}{l}r \\ s\end{array}\right)$ is a vector of quadratic polynomials without constant term. But then it follows that every vector in $P$ has polynomials without constant term. This contradicts Lemma 5.0.4. 


\section{REFERENCES}

[1] Why Vector Bundles, Daniel Chan

https : //www . youtube. com/watch?v=7RGFP JSY ${ }_{L} U$

[2] Irving Kaplansky. [Projective Modules]. Annals of Mathematics, 2nd Series, Volume 68: 372-377, Princeton University Mathematics Department, 1958.

[3] W. Keith Nicholson. Introduction to Abstract Algebra. John Wiley Sons ,Inc., New Jersey, 2012.

[4] Jason Polak. Provjective Modules over Local Rings are Free. Aleph Zero Categorical, 2011-2020.

https ://blog.jpolak.org/?p=363

[5] Joseph J. Rotman. Advanced Modern Algebra. Prentice Hall, 2003.

[6] R. G. Swan, Vector bundles and projective modules, Trans. Amer. Math. Soc. 105 (1962), 264-277.

[7] Charles A. Weibel. The K-Book: An Introduction to Algebraic K-theory. American Mathematical Society, Rhode Island, 2013.

[8] Algebraic K-theory https://en.wikipedia.org/wiki/Algebraic ${ }_{K}$ - theory 